\title{
Can patients with congenitally corrected transposition of the great arteries live better electrically?
}

\author{
Barbara J. Deal, MD
}

\footnotetext{
From the Cardiology Division, Ann \& Robert H. Lurie Children's Hospital, and Feinberg School of Medicine, Northwestern University, Chicago, Ill.

Disclosures: Author has nothing to disclose with regard to commercial support.

Received for publication Sept 2, 2015; accepted for publication Sept 2, 2015; available ahead of print Oct 15, 2015.

Address for reprints: Barbara J. Deal, MD, Cardiology Division, Ann \& Robert H. Lurie Children's Hospital, and Feinberg School of Medicine, Northwestern University, 225 E Chicago Ave, Chicago, IL 60611 (E-mail: bdeal@luriechildrens.org).

J Thorac Cardiovasc Surg 2016;151:140-2

$0022-5223 / \$ 36.00$

Copyright $($ c 2016 by The American Association for Thoracic Surgery

http://dx.doi.org/10.1016/j.jtcvs.2015.09.017
}

In congenitally corrected transposition of the great arteries (ccTGA), the combination of atrioventricular and ventriculoarterial discordance results in an anatomic right ventricle in the subaortic position. This is in frequent association with abnormal tricuspid valve morphology, ventricular septal defects, pulmonic stenosis/atresia, and progressive atrioventricular conduction disorders. Development of significant ventricular dysfunction and tricuspid regurgitation over time results in congestive heart failure and decreased life expectancy. In the multicenter long-term outcomes study of ccTGA by Graham and colleagues published in 2000, ${ }^{1}$ by age 45 years more than two-thirds of patients had clinical congestive heart failure. Furthermore, the risk of congestive heart failure increased with advancing age, associated cardiac anomalies, prior cardiac surgery, tricuspid regurgitation, arrhythmias, and pacemaker implantation. Strategies to minimize late ventricular dysfunction and improve survival have led to a transition from physiologic repair of associated defects in ccTGA leaving a systemic right ventricle, to anatomic repairs with atrial redirection surgery combined with either a Rastelli procedure or arterial switch, resulting in a systemic left ventricle. Although early outcomes are reported to be favorable, the durability of this strategy deserves our attention.

In this issue of the Journal, Hofferberth and colleagues ${ }^{2}$ from Boston Children's Hospital report their evolving and innovative experience with the influence of pacing on ventricular dysfunction in patients with ccTGA and atrioventricular (AV) block. Between 1993 and 2014, 53 patients with ccTGA received epicardial dual-chamber pacemakers for AV block at a median age of 1.8 years, and the authors report median follow-up of 3.7 years (range, 4 days22.5 years). Anatomic repairs were ultimately performed in 52 out of 53 patients; pacemakers were implanted before anatomic repair in 17 patients $(32 \%)$ and at the time of anatomic repair or postoperatively in 36 patients $(68 \%)$. Pacemakers were initially univentricular in 42 patients (79\%), with primary biventricular pacing performed in 11 patients $(21 \%)$. Upgrades of univentricular pacing systems

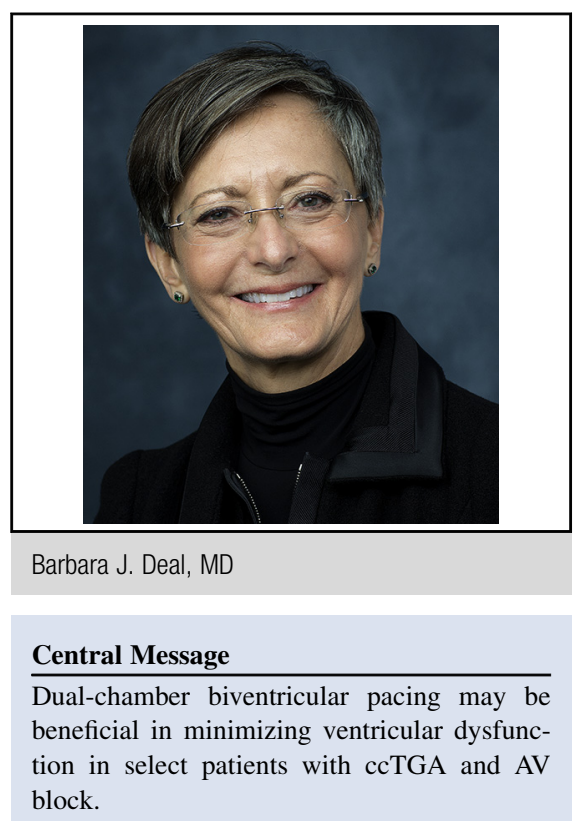

See Article page 131

See Editorial page 15 .

were performed in 17 patients $(40 \%)$ after an interval of approximately 4.5 years. The indication for biventricular pacing upgrade was ventricular dysfunction in 14 out of 17 patients and was performed before anatomic repair in 3 patients and during or following anatomic repair in 11 patients. Elective upgrade to biventricular pacing at the time of anatomic repair was performed in 3 patients without significant ventricular dysfunction. Primary biventricular pacing systems were implanted in 11 patients without evidence of ventricular dysfunction (at the time of anatomic repair in 8 patients and before anatomic repair in 3 patients). Of note, the median QRS duration in patients with primary biventricular pacing was $82 \mathrm{~ms}$ (range, 68-132 ms), and increased to a median of $111 \mathrm{~ms}$ with pacing (range, 102-254 ms). Of these 11 patients with narrow QRS durations and normal ventricular function receiving primary biventricular pacing systems, none developed ventricular dysfunction during short-term follow-up. Among the 14 patients receiving upgrades to biventricular pacing for ventricular dysfunction, half demonstrated improvement in ventricular function. Based on these results, the authors recommend primary biventricular pacing for patients with ccTGA and new-onset AV block to limit the potential development of ventricular 
dysfunction and preemptive placement of biventricular pacing leads at the time of anatomic repairs.

This is an important study in the efforts to minimize longterm ventricular dysfunction in patients with ccTGA who develop AV block. In addition to the negative effect of pacing on ventricular synchrony and contractility, ventricular pacing after anatomic repair is complicated by limited transvenous access to the systemic left ventricle, and the relationship of the coronary sinus to the now subpulmonary right ventricle. Efforts to minimize ventricular dysynchrony created by chronic subpulmonary ventricular pacing are essential. Two key issues highlighted by these data are the incidence of AV block related to anatomic repair and the utility of biventricular pacing in this setting.

The need for chronic ventricular pacing is identified in about $38 \%$ of adult patients with ccTGA by age 40 years, and the need for pacing is associated with worsening of ventricular function in multiple reports. ${ }^{1,3}$ The risk of surgical AV block related to anatomic repairs of ccTGA is variously reported between $3 \%$ and $16 \%$ of patients, ${ }^{4-8}$ and based on a meta-analysis of surgical strategies in ccTGA, ${ }^{9}$ is higher in patients undergoing the arterial switch operation (12\%) versus the Rastelli (4\%). The current report by Hofferberth and colleagues ${ }^{2}$ does not provide us with the number of patients undergoing anatomic repairs during this time frame to determine the incidence of surgical AV block at their center. However, Bautista-Hernandez and colleagues reported in $2014^{10}$ the outcomes of 106 patients undergoing anatomic repair for ccTGA in Boston between 1992 and 2014: Preoperative AV block was present in $14 \%$, with operative or postoperative AV block in $27 \%$. In that study,${ }^{10}$ median age at surgery was 1.2 years with mean follow-up of 5.2 years. During follow-up $38 \%$ of patients required chronic pacing. ${ }^{10}$ Pacing rates of $38 \%$ are similar to the rates reported in patients with ccTGA undergoing physiologic repairs during their fourth decade of life. ${ }^{1,3}$ These findings point out the unfortunate balancing act of the benefits of circulation with a systemic left ventricle at the cost of additional years and perhaps decades of ventricular pacing.

Resynchronization therapy is indicated in adult populations with ventricular ejection fractions below 30\% to $35 \%$, in the presence of QRS prolongation $\geq 120 \mathrm{~ms}$ and symptomatic heart failure despite optimal medical therapy. Similar guidelines for young patients with congenital heart disease do not yet exist. Efforts to demonstrate resynchronization efficacy for a failing ventricle following repair of congenital heart disease are increasing, but challenged by the difficulties in adequately assessing ventricular function and small numbers of patients. A recent study by Yeo and colleagues ${ }^{11}$ evaluated 59 adults with ccTGA using serial sophisticated echocardiographic imaging: 30 without pacing, 22 with univentricular subpulmonary left ventricle pacing, and 7 with biventricular pacing. Multivariate analysis demonstrated that pacing was an independent predictor of right

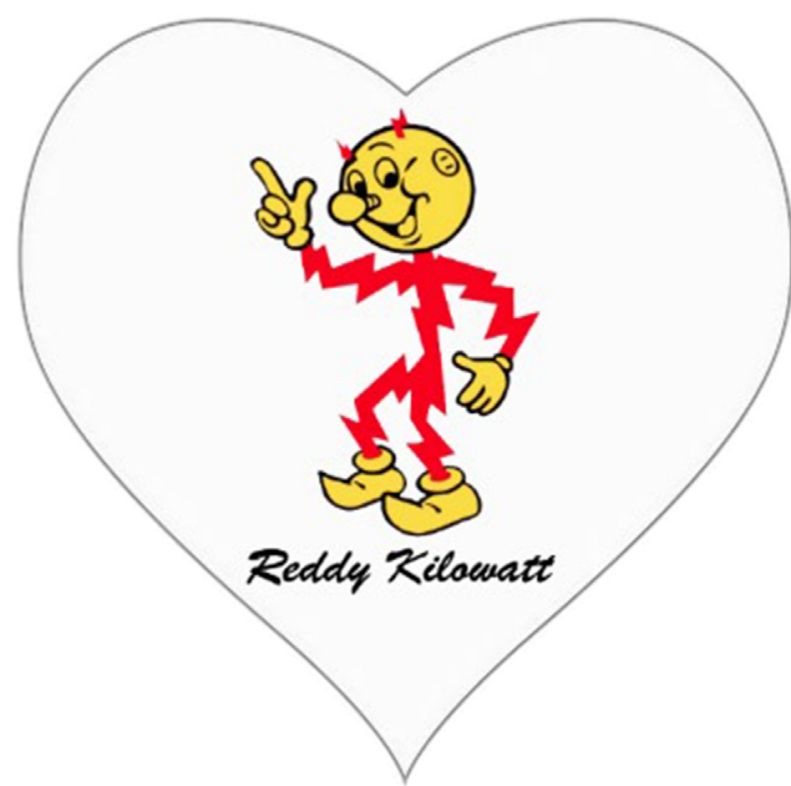

FIGURE 1. Reddy Kilowatt, the image used in conjunction with the 1950s advertising slogan, "Live better electrically."

ventricle dilation and deteriorating function, with unpaced patients showing no interval decline in ventricular function. ${ }^{11}$ Their study ${ }^{11}$ endorsed the use of biventricular pacing rather than univentricular pacing in this population. Cecchin and colleagues ${ }^{12}$ demonstrated variable degrees of improvement in ejection fraction with resynchronization therapy in 46 adolescents and young adults with QRS prolongation following repair of congenital heart disease. However, in the subset of patients with cardiac resynchronization therapy performed soon after cardiac surgery, the acute improvements in ejection fraction were not maintained after 3 months. Studies on biventricular pacing were all performed in patients with preexisting marked prolongation in QRS intervals and ventricular dysfunction, and emphasized the importance of patient selection, optimal lead placement, and postimplantation assessment of programming. The extension of biventricular pacing to patients without QRS prolongation was recently evaluated in a multicenter randomized trial of 809 adults with congestive heart failure, low ejection fractions, and narrow QRS duration $(<130 \mathrm{~ms})$. $^{13}$ The Echocardiography Guided Cardiac Resynchronization Therapy study, as it was known, was stopped prematurely due to findings that resynchronization therapy in patients with a narrow QRS duration may increase mortality. ${ }^{13}$ Therefore, the uniform application of biventricular pacing in ccTGA patients without significant QRS prolongation, as reported in the 11 primary biventricular pacing patients described by Hofferberth and colleagues, ${ }^{2}$ is of concern. Is it possible that univentricular pacing of the systemic ventricle under these conditions would be equally effective?

Multivariate analysis of pacing site selections, QRS durations prior and following pacing or resynchronization, and 
more sophisticated measures of ventricular function utilizing tissue Doppler or speckle tracking will be needed to determine the efficacy of biventricular pacing in ccTGA. An analysis of clinical and pacing characteristics among responders and nonresponders to biventricular pacing will be particularly important. The consensus guidelines from the Heart Rhythm Society for reporting outcomes of interventions for atrial fibrillation have been standardized, and include a minimum follow-up duration of 1 year, with serial evaluations at 3 months and every 6 months for 2 years. Given the relatively small numbers of patients internationally undergoing anatomic repairs for ccTGA, and the need for transparency in counseling families regarding anticipated outcomes, similar efforts to standardize measures of efficacy are more crucial in congenital heart disease populations.

"Living better electrically" was a popular advertising slogan in the late 1950s to encourage the widespread use of electrical appliances in the home as well as energysaving measures to improve the quality of modern life (Figure 1). Given the high risk of developing conduction disorders, tachyarrhythmias, and heart failure following complicated surgical repairs of congenital heart disease, optimizing outcomes using adjunctive device therapy should have a similar expectation of improving quality of life. Prophylactic placement of biventricular epicardial leads or early biventricular pacing is an intellectually appealing strategy that warrants careful adoption and uniform reporting. Efforts to reduce the incidence of operative $\mathrm{AV}$ block are another component of the energy-saving measures that are recommended. Efforts to minimize the late development of ventricular dysfunction in ccTGA by performing anatomic repairs pose unique obstacles, and careful serial evaluations of evidence to determine the long-term benefits of this approach are needed.

\section{References}

1. Graham TP, Bernard YD, Mellen BG, Celermajer D, Baumgartner H, Cetta F, et al. Long term outcome in congenitally corrected transposition of the great arteries: a multi-institutional study. J Am Coll Cardiol. 2000; 36:255-61.

2. Hofferberth SC, Alexander ME, Mah DY, Bautista-Hernandez V, del Nido PJ, Fynn-Thompson F. Impact of pacing on systemic ventricular function in L-transposition of the great arteries. J Thorac Cardiovasc Surgery. 2016;151:131-9.

3. Dobson R, Danton M, Nicola W, Hamish W. The natural and unnatural history of the systemic right ventricle in adult survivors. J Thorac Cardiovasc Surg. 2013; 145:1493-503.

4. Duncan BW, Mee RBB. Management of the failing systemic right ventricle. Semin Thorac Cardiovasc Surg. 2005;17:160-9.

5. Sharma R, Talwar S, Marwah A, Shah S, Maheshwari S, Suresh P, et al. Anatomic repair for congenitally corrected transposition of the great arteries. $J$ Thorac Cardiovasc Surg. 2009; 137:404-12.

6. Gaies MG, Goldberg CS, Ohye RG, Devaney EJ, Hirsch JC, Bove EL. Early and intermediate outcome after anatomic repair of congenitally corrected transposition of the great arteries. Ann Thorac Surg. 2009;88:1952-60.

7. Lim HG, Lee JR, Kim YJ, Park YH, Jun TG, Kim WH, et al. Outcomes of biventricular repair for congenitally corrected transposition of the great arteries. Ann Thorac Surg. 2010;89:159-67.

8. Murtuza B, Barron DJ, Stumper O, Stickley J, Eaton D, Jones TJ, et al. Anatomic repair for congenitally corrected transposition of the great arteries: a single-institution 19 year experience. J Thorac Cardiovasc Surg. 2011;142: 1348-57.

9. Alghamdi AA, McCrindle BW, Van Arsdell GS. Physiologic versus anatomic repair of congenitally corrected transposition of the great arteries: metaanalysis of individual patient data. Ann Thorac Surg. 2006;81:1529-35.

10. Bautista-Hernandez V, Myers PO, Cecchin F, Marx GR, del Nido PJ. Late left ventricular dysfunction after anatomic repair of congenitally corrected transposition of the great arteries. J Thorac Cardiovasc Surg. 2014;148: 254-8.

11. Yeo WT, Jarman JWE, Li W, Gatzoulis MA, Wong T. Adverse impact of chronic subpulmonary left ventricular pacing on systemic right ventricular function in patients with congenitally corrected transposition of the great arteries. Int J Card. 2014;171:184-91.

12. Cecchin F, Frangini PA, Brown DW, Fynn-Thompson F, Alexander ME, Triedman JK, et al. Cardiac resynchronization therapy and multisite pacing in pediatrics and congenital heart disease: Five years' experience in a single institution. J Cardiovasc Electrophysiol. 2009;20:58-65.

13. Ruschitzka F, Abraham WT, Singh JP, Bax JJ, Borer JS, Brugada J, et al. Cardiacresynchronization therapy in heart failure with a narrow QRS complex. $N$ Engl J Med. 2013;369:1395-405. 